\title{
Non-Hermitian Random Matrices and the Calogero-Sutherland Model
}

\author{
Pragya Shukla \\ Department of Physics, Indian Institute of Technology, Kharagpur, India
}

(Received 30 March 2001; published 23 October 2001)

\begin{abstract}
We study the statistical properties of the eigenvalues of non-Hermitian operators associated with the dissipative complex systems. By considering the Gaussian ensembles of such operators, a hierarchical relation between the correlators is obtained. Further, the eigenvalues are found to behave like particles moving on a complex plane under two-body (inverse square) and three-body interactions and there seems to underlie a deep connection and universality in the spectral behavior of different complex systems.
\end{abstract}

DOI: 10.1103/PhysRevLett.87.194102

The random non-Hermitian $(\mathrm{NH})$ operators play a significant role in the dynamics of a variety of complex systems, e.g., dissipative quantum systems [1,2], chaotic quantum scattering [3], neural network dynamics [4], statistical mechanics of flux lines in superconductors with columnar disorder [5], classical diffusion in random media [6], and biological growth problems [7]. A detailed knowledge of the statistical properties of their eigenvalues and eigenvectors therefore is very desirable. However, so far, the information is available only for a few specific cases, e.g., $[1,2,8,9]$.

The complicated nature of the interactions (or a lack of detailed information about them) in a system introduces a degree of randomness in the matrix representation of the associated operators. The indeterminacy of the interactions permits each matrix element to be described only by a distribution of possible values, resulting in a random matrix. However, the choice of a suitable random matrix model for an operator of a complex system is very sensitive to the nature of its complexity. The statistical spectral analysis of different complex systems requires, therefore, a thorough probing of a wide range of random matrix ensembles which is not an easy task. It is highly desirable, if possible, to find a common mathematical formulation for various systems where the information about the system enters through a parameter. The possibility of such a formulation has already been shown for the Hermitian operators [10]. This encourages us to seek the same for non-Hermitian operators also. We explore the nonHermitian random matrix ensembles (NHRE) with a Gaussian distribution and obtain the formulation using exact analytical methods. This is achieved by showing that the eigenvalue distributions of various NHREs appear as nonequilibrium stages of a Brownian-type diffusion process. Here the eigenvalues evolve with respect to a parameter related to the complexity of the system associated with the NHRE. The solution of the diffusion equation for a given value of the parameter gives, therefore, the distribution of the eigenvalues for the corresponding system. By using the diffusion equation, we also obtain the hierarchic relations among eigenvalue correlations.

The connection of various NHREs through a diffusion process can further be used to reveal a very interesting fea-
PACS numbers: 05.45. $-\mathrm{a}, 03.65 .-\mathrm{w}, 05.40 .-\mathrm{a}, 82.40 .-\mathrm{g}$

ture about their eigenvalue dynamics in the following way. A suitable transformation can reduce the diffusion equation to the Schrödinger equation of a classically integrable Hamiltonian, thereby mapping the eigenvalue distribution of a general NHRE to a nonstationary state of the Hamiltonian. The latter, a variant of the CalogeroSutherland (CS) Hamiltonian in two dimensions, is a generator of the dynamics of $N$ particles interacting via long-range two-body and three-body interactions and confined by a harmonic oscillator potential $[11,12]$. The information about the spectral correlators of a given $\mathrm{NH}$ system can therefore be obtained from the particle correlations of the CS system. As is well known, the CS Hamiltonian is an integrable system with particles evolving in an ordered way with respect to time; this indicates a strong correlation between various particle states at different times. Our mapping thus implies that the eigenvalues evolve in a highly ordered, correlated way as the degree or the nature of the complexity changes. This would also indicate a strong correlation between the statistical nature of the eigenvalues of different complex systems. Note that another study has already indicated the universality in the eigenvalue statistics of the operators in the regime of weak non-Hermiticity [13].

We consider an ensemble of $N \times N$ non-Hermitian matrices $H$ defined by a Gaussian measure $\tilde{\rho}(H, y, x)$, where $\quad \tilde{\rho} \propto \exp \left[-\sum_{s=1}^{\beta} \sum_{k, l}\left(y_{k l ; s} H_{k l ; s}^{2}+x_{k l ; s} H_{k l ; s} \times\right.\right.$ $\left.\left.H_{l k ; s}\right)\right]=C \rho(H, y, x)$ with $C$ as the normalization constant, and $y$ and $x$ as sets of the variances and covariances of various matrix elements. Here the subscript $s$ on a variable refers to one of its components, i.e., real $(s=1)$ or imaginary $(s=2)$ part, with $\beta$ as the total number of the components. The above choice of $\rho$ is made so as to include a large class of NHRE.

A non-Hermitian matrix can be diagonalized by a transformation of the type $\Lambda=\mathrm{UHV}$ with $\Lambda$ as the matrix of eigenvalues $\lambda_{j}$, and $U$ and $V$ as the left and right eigenvector matrices, respectively. Let us first consider the case of an ensemble of non-Hermitian complex matrices $(\beta=2)$. Here the eigenvalues $\lambda_{j} \equiv \sum_{r=1}^{2}(i)^{r-1} \lambda_{j r}$, in general $(r$ referring to the components of the eigenvalues), are distributed over an area in the complex plane. Let $\tilde{P}(z, y, x)$ be the probability of finding eigenvalues $\lambda_{i}$ of $H$ between 
$z_{i}$ and $z_{i}+d z_{i}$ for given sets $y$ and $x$,

$$
\tilde{P}(z, y, x)=C \int f\left(z, z^{*}\right) \rho(H, y, x) d H
$$

with $z \equiv\left\{z_{i}\right\}$ and $f\left(z, z^{*}\right)=\prod_{i=1}^{N} \delta\left(z_{i}-\lambda_{i}\right) \delta\left(z_{i}^{*}-\right.$ $\left.\lambda_{i}^{*}\right)$.

The degree of difficulty associated with solving the integral Eq. (1) motivates us to seek another route. We attempt to obtain an evolution equation, in a well-known form, for $P=\tilde{P} / C$ due to changing distribution parameters. (The prior familiarity with the equation can then be used to obtain the information about its solution $P)$. For this purpose, we consider a combination of the parametric derivatives of $P$, namely, the sum $S, S \equiv$ $\sum_{s=1}^{\beta} \sum_{k, l}\left[A_{k l ; s} \frac{\partial P}{\partial y_{l ;: s}}+B_{k l ; s} \frac{\partial P}{\partial x_{k l ; s}}\right]$ and express it in terms of the eigenvalue derivatives of $P$. Here $A_{k l ; s}=y_{k l ; s}[\gamma+$ $\left.2(-1)^{s} x_{l k ; s}\right] \quad$ and $\quad B_{k l ; s}=\left[\gamma x_{k l ; s}+(-1)^{s} x_{k l ; s} x_{l k ; s}+\right.$ $\left.(-1)^{s} y_{k l ; s} y_{l k ; s}\right]$ (with $\gamma$ as an arbitrary parameter and, as shown later, its arbitrary value gives the freedom to choose the end of the evolution). This would require a knowledge of the rates of change of the eigenvalues as well as the eigenvectors due to a small change in the matrix element $H_{k l}$, given as follows:

$$
\begin{aligned}
\frac{\partial \lambda_{n}}{\partial H_{k l ; s}} & =i^{s-1} U_{n k} V_{l n} ; \\
\sum_{k, l ; s} \frac{\partial \lambda_{n}}{\partial H_{k l ; s}} H_{k l ; s} & =\lambda_{n}, \\
\sum_{k, l ; s}(-1)^{s-1} \frac{\partial \lambda_{n}}{\partial H_{k l ; s}} \frac{\partial \lambda_{m}}{\partial H_{l k ; s}} & =\beta \delta_{m n}, \\
\sum_{k, l ; s}(-1)^{s-1} \frac{\partial^{2} \lambda_{n}}{\partial H_{k l ; s} H_{l k ; s}} & =\sum_{m} \frac{2 \beta}{\lambda_{n}-\lambda_{m}}, \\
\frac{\partial U_{n r}}{\partial H_{k l ; s}} & =\sum_{m \neq n} \frac{U_{n k}}{\lambda_{n}-\lambda_{m}} \frac{\partial \lambda_{m}}{\partial H_{r l ; s}}, \\
\frac{\partial V_{r n}}{\partial H_{k l ; s}} & =\sum_{m \neq n} \frac{V_{l n}}{\lambda_{n}-\lambda_{m}} \frac{\partial \lambda_{m}}{\partial H_{k r ; s}} .
\end{aligned}
$$

The parametric dependence of $P$ in sum $S$ enters only through $\rho$ and, as $\frac{\partial \rho}{\partial y_{k l ; s}}=-H_{k l ; s}^{2} \rho, \frac{\partial \rho}{\partial x_{k l ; s}}=-H_{k l ; s} \times$ $H_{l k ; s} \rho$, and $\frac{\partial \rho}{\partial H_{k l ; s}}=-2\left(y_{k l ; s} H_{k l ; s}+x_{k l ; s} H_{l k ; s}\right) \quad$ with $\frac{\partial f}{\partial H_{k l ; s}}=-2 \sum_{r} \frac{\partial \lambda_{n r}}{\partial H_{k l ; s}} \frac{\partial f}{\partial z_{n r}}$, the sum $S$ can be rewritten as

$$
S=\sum_{s=1}^{\beta} \sum_{k, l}\left[\gamma+(-1)^{s} x_{k l ; s}\right] I_{k l ; s}+G-C_{1} P
$$

where $I_{k l ; s}=\sum_{r, n} \frac{\partial}{\partial z_{n r}} \int f \frac{\partial \lambda_{n r}}{\partial H_{k l ; s}} H_{k l ; s} \rho d H, G=\sum_{k, l ; s} \times$ $(-1)^{s}\left[y_{k l ; s} y_{l k ; s} \frac{\partial P}{\partial x_{k l ; s}}+x_{k l ; s} y_{l k ; s} \frac{\partial P}{\partial y_{l k ; s}}\right]$, and $C_{1}=(1 / 2) \times$ $\sum_{k, l ; s}\left[\gamma+(-1)^{s} x_{k l ; s}\right]$. Further, by using Eqs. (2), one can show that $\sum_{k, l ; s} I_{k l ; s}=\sum_{n, r} \frac{\partial}{\partial z_{n r}}\left(z_{n r} P\right)$ and $\sum_{k, l ; s}(-1)^{s} x_{k l ; s} I_{k l ; s}=\frac{\partial^{2} P}{\partial z_{n r}^{2}}-2 \frac{\partial}{\partial z_{n r}}\left(\frac{\partial \ln |\Delta(z)|}{\partial z_{n r}} P\right)-G$; here $\Delta_{N}(z)=\prod_{j<k}^{N}\left(z_{j}-z_{k}\right)$. A substitution of these equalities in Eq. (3), followed by a comparison of the so-obtained form of $S$ with its original definition, gives now a relation between the parametric and eigenvalue derivatives of $P$,

$$
\begin{aligned}
& \sum_{s=1}^{\beta} \sum_{k, l}\left[A_{k l ; s} \frac{\partial P}{\partial y_{l k ; s}}+B_{k l ; s} \frac{\partial P}{\partial x_{k l ; s}}\right]+C_{1} P \\
& =\sum_{r=1}^{2} \sum_{n=1}^{N} \frac{\partial}{\partial z_{n r}}\left[\frac{\partial}{\partial z_{n r}}-\beta \frac{\partial \ln \left|\Delta_{N}(z)\right|}{\partial z_{n r}}+\gamma z_{n r}\right] P .
\end{aligned}
$$

However, it is possible to define a parameter $Y$, a function of all $y_{k l ; s}$ and $x_{k l ; s}$, such that the sum on the left-hand side of the above equation can be reduced to a derivative of $P$ with respect to a single parameter $Y$, with $P(z, y, x)=$ $P(z, Y)$. Obviously, $Y$ should satisfy the condition that

$$
\sum_{s=1}^{\beta} \sum_{k, l}\left[A_{k l ; s} \frac{\partial P}{\partial y_{l k ; s}}+B_{k l ; s} \frac{\partial P}{\partial x_{k l ; s}}\right]=\frac{\partial P}{\partial Y} .
$$

As $\frac{\partial P}{\partial r}=\frac{\partial P}{\partial Y} \frac{\partial Y}{\partial r}, r$ taken from sets $y$ or $x$, the above condition can be rewritten as $\sum_{s=1}^{\beta} \sum_{k, l}\left[A_{k l ; s} \frac{\partial Y}{\partial y_{l k ; s}}+B_{k l ; s} \times\right.$ $\left.\frac{\partial Y}{\partial x_{k l ;}}\right]=1$. The form of $Y$, fulfilling the condition, can therefore be obtained by solving the following equations [10] (for all $k l$, and $s$ values): $\frac{d y_{k l ; s}}{A_{k l ; s}}=\frac{d x_{k l ; s}}{B_{k i s}}=\frac{d Y}{1}$. The solution $Y$ turns out to be $Y=\left(1 / \beta N^{2}\right) \sum_{k, l ; s} \times$ $F\left(y_{k l ; s}\right)+Y_{0}$ with $Y_{0}$ given by the initial conditions. Here $F\left(y_{k l ; s}\right)=(-1)^{s} \int d y_{k l ; s}\left(y_{k l ; s} \sqrt{W}\right)^{-1}=-\ln \{2 \times$ $\left.\left[\gamma^{2}+2(-1)^{s} \tilde{c}_{k l ; s} y_{k l ; s}+\gamma \sqrt{W}\right] / y_{k l ; s}\right\}$ with $W=\gamma^{2}+$ $4 y_{k l ; s}\left[c_{k l ; s} y_{k l ; s}+(-1)^{s} \tilde{c}_{k l ; s}\right]$ and constants $c_{k l ; s}$ and $\tilde{c}_{k l ; s}$ given by relations $y_{l k ; s}=c_{k l ; s} y_{k l ; s}$ and $x_{k l ; s}^{2}+$ $(-1)^{s} \gamma x_{k l ; s}-c_{k l ; s} y_{k l ; s}^{2}-(-1)^{s} \tilde{c}_{k l ; s} y_{k l ; s}=0$. Further, since all $y_{k l ; s}$ and $x_{k l ; s}$ are indicators of the complexity of the system, $Y$ can be termed as the complexity parameter. Now, by defining $P_{1}=C_{2} P$ with $C_{2}=e^{\int C_{1} d Y}$, Eq. (4) can be rewritten as the equation governing the evolution of eigenvalues in terms of the parameter $Y$,

$$
\begin{aligned}
\frac{\partial P_{1}}{\partial Y}= & \sum_{r=1}^{2} \sum_{n=1}^{N} \frac{\partial}{\partial z_{n r}}\left[\frac{\partial}{\partial z_{n r}}-\beta \frac{\partial \ln \left|\Delta_{N}(z)\right|}{\partial z_{n r}}+\gamma z_{n r}\right] \\
& \times P_{1},
\end{aligned}
$$

where $\beta=2$ and $P_{1}$ is related to the normalized distribution by $\tilde{P}=C P_{1} / C_{2}$. Note the analogy of the above equation to that of the Hermitian case [10] but the evolution is now occurring on a complex plane.

The steady state of Eq. (6), $P_{s} \equiv\left|Q_{N}\right|^{2}=\prod_{j<k} \times$ $\left|\Delta_{N}(z)\right|^{2} e^{-\gamma / 2 \sum_{k}\left|z_{k}\right|^{2}}, \quad$ corresponds to $\frac{\partial P}{\partial Y} \rightarrow 0$ and $Y \rightarrow \infty$ which is possible when almost all $F\left(y_{k l ; s}\right) \rightarrow \infty$ or, equivalently, $\tilde{c}_{k l ; s}^{2}=\gamma^{2} c_{k l ; s}$. The latter gives the condition on $y_{k l ; s}$ and $x_{k l ; s}$ for a steady state to occur. A choice of almost all $y_{k l ; s} \rightarrow N /\left(1-\tau^{2}\right)$ and $x_{k l ; s} \rightarrow$ $(-1)^{s-1} N \tau /\left(1-\tau^{2}\right)$ with $\gamma=1$ fulfills the condition for $\tau \rightarrow 0, \pm 1$ and can therefore lead to three different types of steady states, namely, Ginibre $(\tau=0)$, Gaussian unitary ensemble (GUE) $(\tau=1)$, and the ensemble of complex symmetric matrices (GASE, $\tau=-1$ ). Here the distribution $P_{s}$ represents all three cases and, in each case, agrees well with the already known distributions for 
Ginibre (GBE) $\left(z_{k}\right.$ as complex eigenvalues), GUE ( $z_{k}$ as real eigenvalues), and GASE (thus as eigenvalues in equal and opposite pairs).

Equation (6) describes a transition from a given initial ensemble (with $Y=Y_{0}$ ) to either GBE, GUE, or GASE with $Y-Y_{0}$ as the transition parameter. The nonequilibrium states of these transitions, given by nonzero finite values of $Y-Y_{0}$, are various ensembles of the complex matrices corresponding to varying values of $y_{k l}$ 's and $x_{k l}$ 's, thus modeling different complex systems. Note that Eq. (6) for $P_{1} \equiv P_{1}\left(\mu, Y \mid \mu_{0}, Y_{0}\right)$ has been obtained for arbitrary initial conditions, say, $P_{1}\left(\mu_{0}, Y_{0}\right)$; the distribution $P_{1}(\mu, Y)=\int P_{1}\left(\mu, Y \mid \mu_{0}, Y_{0}\right) P_{1}\left(\mu_{0}, Y_{0}\right) d \mu_{0}$ of a given NHRE can therefore be found by solving Eq. (6) by using a convenient initial ensemble. Just as in the Hermitian case, the "convenience" depends on mathematical tractability of the integrals as well as on involved physics [10].

The case of non-Hermitian real matrices $(\beta=1)$ can similarly be treated. Here eigenvalues are either real or form complex conjugate pairs, and therefore, if $U_{n}$ is an eigenvector corresponding to the eigenvalue $\lambda_{n}$, its complex conjugate $U_{n}^{*}$ will correspond to the eigenvalue $\lambda_{n}^{*}$. Consider the case with $L$ real and $M$ complex conjugate pairs of the eigenvalues with $N=L+2 M$. The rates of change of the eigenvalues and the eigenvectors are still given by Eqs. (2) with $H_{k l ; 1} \equiv H_{k l}$. The distribution $P$ in this case can be described by $P=\int \prod_{j=1}^{N} \times$ $f\left(z, z^{*}\right) g\left(z, z^{*}\right) \rho(H) d H \quad$ with $\quad f\left(z, z^{*}\right)=\prod_{j=1}^{L} \delta\left(z_{j}-\right.$ $\left.\lambda_{j}\right) \delta\left(z_{j}-\lambda_{j}^{*}\right), \quad g\left(z, z^{*}\right)=\prod_{j=L+1}^{L+M} \delta\left(z_{j}-\lambda_{j}\right) \delta\left(z_{j}^{*}-\right.$ $\left.\lambda_{j+M}\right) \delta\left(z_{j+M}-\lambda_{j}^{*}\right) \delta\left(z_{j+M}^{*}-\lambda_{j+M}^{*}\right)$. (As is obvious, here the first $L$ eigenvalues are real and rest of them are complex conjugate pairs.) Proceeding similarly as for the complex case, by using Eqs. (2) and equalities $\frac{\partial f g}{\partial H_{k l}}=-\sum_{n=1}^{L+2 M} \frac{\partial f g}{\partial z_{n}} \frac{\partial \lambda_{n}}{\partial H_{k l}}, \quad \frac{\partial^{2} f g}{\partial H_{k l} H_{l k}}=-4 \sum_{n=1}^{L+2 M} \frac{\partial}{\partial z_{n}} \times$ $\left(\frac{\partial f g}{\partial z_{n}}+\sum_{m \neq n} \frac{f g}{\lambda_{n / m}-\lambda_{n}}\right)$, one obtains

$$
\frac{\partial P_{1}}{\partial Y}=\sum_{n=1}^{L+2 M} \frac{\partial}{\partial z_{n}}\left[\frac{\partial}{\partial z_{n}}-\beta \frac{\partial \ln |\Delta(z)|}{\partial z_{n}}+\gamma z_{n}\right] P_{1},
$$

where $Y$ is still given by Eq. (5) with $\beta=1\left(y_{k l ; 1} \equiv y_{k l}\right.$ and $\left.x_{k l ; 1} \equiv x_{k l}\right) ; \quad Y=\frac{1}{N^{2}} \sum_{k, l} F\left(y_{k l}\right)$ with $F\left(y_{k l}\right)=$ $\int d y_{k l}\left(y_{k l} \sqrt{W}\right)^{-1}=-\ln \left[2\left(\gamma^{2}-2 \tilde{c}_{k l} y_{k l}+\gamma \sqrt{W}\right) / y_{k l}\right]$, $W=\gamma^{2}+4 y_{k l}\left(c_{k l} y_{k l}-\tilde{c}_{k l}\right)$, and $c_{k l}, \tilde{c}_{k l}$ given by relations $y_{l k}=c_{k l} y_{k l}$ and $x_{k l}^{2}-\gamma x_{k l}-c_{k l} y_{k l}^{2}+\tilde{c}_{k l} y_{k l}=0$. The steady state again occurs for $Y \rightarrow \infty$ where the corresponding solution of Eq. (7) is given by $P_{1}(Y \rightarrow \infty)=$ $P_{s}=\left|\Delta_{N}(z)\right|\left[\prod_{i=1}^{N} e^{-\gamma z_{i}^{2}} \operatorname{erfc}\left(z_{i}-z_{i}^{*}\right)\right]^{1 / 2}$. As before, the limit $Y \rightarrow \infty$ can be obtained for $y_{k l} \rightarrow N /\left(1-\tau^{2}\right)$, $x_{k l} \rightarrow N \tau /\left(1-\tau^{2}\right)$, and $\gamma=1$ with $\tau \rightarrow 0, \pm 1$; the steady state is an ensemble of real matrices $(\tau=0)$, a $\operatorname{GOE}(\tau=1)$, and a Gaussian ensemble of real antisymmetric matrices $(\tau=-1)$. The distribution $P(z ; \tau=$ $0, \pm 1)$ is in agreement with the results obtained in [14], for the corresponding ensembles, by a different method.
As in the Hermitian case $[10,15]$, a direct integration of Eq. (6) (over all $z_{i}, i=n+1 \rightarrow N$ ) can be used to obtain the $n$th order density correlator $R_{n}\left(z_{1}, \ldots, z_{n} ; Y\right)$, defined by $R_{n}=\frac{N !}{(N-n) !} \int P(z, Y) d^{2} z_{n+1} \ldots d^{2} z_{N}$ with $d^{2} z_{n} \equiv$ $d z_{n 1} d z_{n 2}$. However, for real applications, it is important to consider the limit $N \rightarrow \infty$ for fixed $n$. To take the limit meaningfully for $R_{1}\left(z_{1}\right)$, a change of variable $z_{1} \rightarrow e$ is required, where $z_{1}=\sqrt{N} e$ [as $R_{1}\left(z_{1}\right) \approx O(N)$ ]. An integration of Eq. (6), followed by the change $z_{1} \rightarrow e$, will give the evolution equation of $R_{1}(e)$ :

$$
\frac{\partial R_{1}}{\partial Y}=\sum_{r=1}^{2} \frac{\partial}{\partial e_{r}}\left(\gamma e_{r}-2 \mathbf{P} \int d^{2} e^{\prime} R_{1}\left(e^{\prime}\right) \frac{e_{r}-e_{r}^{\prime}}{\left|e-e^{\prime}\right|^{2}}\right) R_{1}
$$

( $\mathbf{P}$ as the principal part of the integral). Here the terms containing second order cluster functions and the diffusion term (see [15]) have been neglected, both being $O(N)$ (or more) smaller than other terms. For $n>1$, the correlators should be unfolded before taking the limit $N \rightarrow \infty: \quad R_{n}\left(\zeta_{1}, \ldots, \zeta_{n} ; \Lambda\right)=\lim N \rightarrow \infty \frac{\boldsymbol{R}_{n}\left(z_{1}, \ldots, z_{n} ; Y\right)}{\boldsymbol{R}_{1}\left(z_{1} ; Y\right), \ldots \boldsymbol{R}_{1}\left(z_{n} ; Y\right)}$ with $\zeta=\int^{\zeta} \boldsymbol{R}_{1}^{1 / 2}(z ; Y) d z$ and $\Lambda=\left(Y-Y_{0}\right) R_{1}$. Again, first, Eq. (6) is integrated to get the hierarchic relations among $R_{n}(z)$ and, second the limit $N \rightarrow \infty$ is applied after replacing $R_{n}(z)$ by $R_{n}(\zeta)$. This gives

$$
\frac{\partial R_{n}}{\partial \Lambda}=\sum_{r=1}^{b} \sum_{j=1}^{n} \frac{\partial}{\partial \zeta_{j r}}\left[\frac{\partial}{\partial \zeta_{j r}}\left|\Delta_{n}\right|^{\beta} \frac{R_{n}}{\left|\Delta_{n}\right|^{\beta}}-\beta I\left(z_{j}\right)\right]
$$

with $I\left(\zeta_{j}\right) \equiv \int_{-\infty}^{\infty} d^{2} \zeta_{n+1} R_{n+1} \partial \ln \left|\zeta_{j}-\zeta_{n+1}\right| / \partial \zeta_{j r}$ and $b=2$ (for simplification, $\gamma$ is chosen to be unity). Here the rescaling of parameter $Y$ was required to see the smooth transition in $R_{n}$ [the evolution in $R_{n}(\zeta)$ takes place for finite values of $Y R_{1}$ ]. Equation (8) is obtained by neglecting the linear drift of the eigenvalues which is dominated, by a factor $R_{1}$, by their diffusion and mutual repulsion. In fact, the linear restoring force, responsible for the global behavior of the density of levels, is entirely negligible on scales at which local fluctuations occur. On the other hand, the diffusion is ineffective on the global scale (see the equation for $R_{1}$ ). As discussed above, the transition for $R_{n}$ occurs on the scales determined by $Y \approx R_{1}^{-1}$, while, for $R_{1}$, the corresponding scale is given by $Y \approx N R_{1}^{-1}$. This indicates a clear separation of the scales of the global and local behavior of the density. The hierarchical equation of correlations for the real asymmetric case can be obtained by integrating Eq. (7) which will again lead to a relation similar to Eq. (8) but now $\beta=1$. Further, for all those $\zeta_{j}$ which correspond to real eigenvalues, the $\sum_{r}$ is to be dropped, with $\zeta_{j r}$ replaced by $\zeta_{j}$.

An alternative route to obtain correlations is by exploiting the connection of Eq. (6) [and Eq. (7)] to the CS Hamiltonian. This can be shown by using the transformation $\Psi=P /\left|Q_{N}\right|^{\beta / 2}$ in Eq. (6) [and Eq. (7)] and reducing it in a form $\frac{\partial \Psi}{\partial Y}=\hat{H} \Psi$ where the "Hamiltonian" $\hat{H}$ turns out to be a variant of the CS Hamiltonian in two dimensions 
(for simplification take $\gamma=1$ ):

$$
\begin{aligned}
\hat{H}= & \sum_{i} \frac{\partial^{2}}{\partial r_{i}^{2}}-g \sum_{i, j ; i<j} \frac{1}{r_{i j}^{2}}-G \sum_{i, j, k ; i<j, i, j \neq k} \frac{\mathbf{r}_{k i} \cdot \mathbf{r}_{k j}}{r_{k i}^{2} r_{k j}^{2}} \\
& -\sum_{i} r_{i}^{2}
\end{aligned}
$$

with $\mathbf{r}_{i} \equiv z_{i}, \mathbf{r}_{k i} \equiv z_{k}-z_{i}$, and $r_{k i} \equiv\left|\mathbf{r}_{k i}\right|$. Here $G=g$ (with $g=1 / 2$ for a NHRE case with all real eigenvalues and $g=2$ for the complex NHRE) and, unlike the complex Hermitian case $(G=0, g=1)$, the inverse square term does not drop out for the complex non-Hermitian case. Further, here the particles are bosons (as $g=G$ ) instead of fermions (the Hermitian case). As $Y \rightarrow \infty$, the particles are in their ground state $\psi_{0}=\prod_{j<k}^{N} \mid \mathbf{r}_{i}-$ $\mathbf{r}_{j} \mid e^{-1 / 2 \sum_{k}\left|r_{k}\right|^{2}}$ with a distribution $\psi_{0}^{2}$; note $\psi_{0}$ gives the correct form for $P(Y \rightarrow \infty)$. The bosonic radial eigenstates and the eigenvalues of the Hamiltonian $\hat{H}$ for the case $g=$ $G$ are well known: $\psi_{n}=\prod_{j<k}^{N}\left|\mathbf{r}_{i}-\mathbf{r}_{j}\right|^{\Lambda} e^{-1 / 2 \sum_{k}\left|r_{k}\right|^{2}} L_{n}$ with $L_{n}$ as Laguerre's polynomial and energy $E_{n}=[4 n+$ $N(N-1) \Lambda+2 N] / 2, \Lambda=\sqrt{G / 2}[12]$. The "state" $\psi$ or $P\left(\mu, Y \mid \mu_{0}, Y_{0}\right)$ can then formally be expressed as a sum over the eigenvalues and eigenfunctions which, on integration over the initial state $P\left(\mu_{0}, Y_{0}\right)$, would lead to the joint probability distribution $P(\mu, Y)$ and thereby static (at a single parameter value) density correlations $R_{n}$. The above correspondence can also be used to map the multiparametric correlations of levels to multitime correlations of the particle positions [10]. Although the explicit calculations of correlations involves technical handling of various integrals and is still an unfinished task, nonetheless our study reveals an important connection. The level correlations of different complex systems need not be studied separately; a thorough probing of the particle correlations of CS-type Hamiltonians will give all the required information. Since the CS system is integrable in nature, the semiclassical techniques can also be very successful for the probing.

The reasons for the correspondence between the Gaussian NHRE and CS Hamiltonian are worth attention. Note that the analogy with a harmonic oscillator-type confining potential in the CS system results from the Gaussian nature of the ensemble. The correspondence with a $1 / r^{2}$ term comes from the mutual repulsion between eigenvalues. The mathematical origin of the latter lies in the transformation from matrix space to eigenvalueeigenvector space which is the same for all the nonHermitian ensembles (belonging to the same symmetry class, irrespective of matrix element distribution). It should be possible, therefore, to map the non-Gaussian NHRE also to a variant of the CS Hamiltonian, although with a different type of confining potential. For $\rho(H) \propto e^{-f(H)}$, the correspondence can be shown by following the similar steps used for the Hermitian case [10]. Also note that the short-range correlations of all the NHREs are dominated by the mutual repulsion, and therefore are expected to be nearly similar within the same symmetry class.

In this paper, we have studied the statistical properties of the eigenvalues of non-Hermitian systems. We find that the distribution of the eigenvalues is governed by a diffusion equation in which system dependence enters only through the evolution parameter $\Lambda \propto Y-Y_{0}$ related to complexity of the system. The spectral correlators for a given NHRE depend only on the associated $\Lambda$ value. It is possible that widely different systems with different values of the distribution parameters may share the same $\Lambda$ value. Such systems will thus have similar statistical features. Furthermore, since the eigenvalue distribution for each complex system appears as a general state of the CS system, any two such states, for example, $\psi\left(Y_{1}\right)$ and $\psi\left(Y_{2}\right)$, being related by the "time" evolution operator $U\left(Y_{2}, Y_{1}\right)$, the eigenvalue distributions of the complex systems corresponding to $Y_{1}$ and $Y_{2}$ will also be connected. This would also reflect in their physical properties based on spectral fluctuations, e.g., conductance (assuming the existence of ergodicity, that is, ensemble averages the same as spectral averages).

The appearance of a CS Hamiltonian is not restricted only to the spectral properties; it has been known also to manifest itself in other properties of complex systems [16]. A detailed investigation of a CS Hamiltonian in arbitrary dimension can therefore give a lot of useful information about a variety of complex systems and is very desirable.

[1] F. Haake et al., Z. Phys. B 88, 359 (1992).

[2] J. Feinberg and A. Zee, Phys. Rev. E 59, 6433 (1999).

[3] Y. V. Fyodorov, and H.-J. Sommers, J. Math Phys. (N.Y.), 38, 1918 (1997).

[4] H. J. Sommers et al., Phys. Rev. Lett. 60, 1895 (1988).

[5] N. Hatano and D. R. Nelson, Phys. Rev. Lett. 77, 570 (1996); K. B. Efetov, Phys. Rev. B 56, 9630 (1997); I. Y. Glodsheild and B. A. Khoruzhenko, Phys. Rev. Lett. 80, 2897 (1998); C. Mudry, B. D. Simons, and A. Altland, Phys. Rev. Lett. 80, 4257 (1998).

[6] J. T. Chalker and Z. J. Wang, Phys. Rev. Lett. 79, 1797 (1997).

[7] D. R. Nelson and N. M. Shnerb, cond-mat/9708071.

[8] J. T. Chalker and B. Mehlig, Phys. Rev. Lett. 81, 3367 (1998).

[9] Y. V. Fyodorov, B. A. Khoruzhenko, and H.-J. Sommers, Phys. Rev. Lett. 79, 557 (1997).

[10] P. Shukla, Phys. Rev. E 62, 2098 (2000).

[11] F. Calogero and C. Marchiero, J. Math. Phys. 14, 182 (1973).

[12] A. Khare and Koushik Ray, Phys. Lett. A 230, 139 (1997).

[13] Y. V. Fyodorov and B. A. Khoruzhenko, Ann. Inst. Henri Poincare Phys. Theor. 68, 449 (1998).

[14] Nils Lehmann and H.-J. Sommers, Phys. Rev. Lett. 67, 941 (1991).

[15] A. Pandey and P. Shukla, J. Phys. A, 24, 3907 (1991).

[16] J. Diejen and L. Vinet, Calogero-Moser-Sutherland Models (Springer, New York, 2000). 